\title{
The mammalian circadian clock and its entrainment by stress and exercise
}

\author{
Yu Tahara $^{1,2} \cdot$ Shinya Aoyama $^{1} \cdot$ Shigenobu Shibata $^{1}$
}

Received: 24 September 2015/Accepted: 7 April 2016/Published online: 15 April 2016

(C) The Physiological Society of Japan and Springer Japan 2016

\begin{abstract}
The mammalian circadian clock regulates daynight fluctuations in various physiological processes. The circadian clock consists of the central clock in the suprachiasmatic nucleus of the hypothalamus and peripheral clocks in peripheral tissues. External environmental cues, including light/dark cycles, food intake, stress, and exercise, provide important information for adjusting clock phases. This review focuses on stress and exercise as potent entrainment signals for both central and peripheral clocks, especially in regard to the timing of stimuli, types of stressors/exercises, and differences in the responses of rodents and humans. We suggest that the common signaling pathways of clock entrainment by stress and exercise involve sympathetic nervous activation and glucocorticoid release. Furthermore, we demonstrate that physiological responses to stress and exercise depend on time of day. Therefore, using exercise to maintain the circadian clock at an appropriate phase and amplitude might be effective for preventing obesity, diabetes, and cardiovascular disease.
\end{abstract}

Y. Tahara and S. Aoyama contributed equally to this study.

Shigenobu Shibata

shibatas@waseda.jp

1 Laboratory of Physiology and Pharmacology, School of Advanced Science and Engineering, Waseda University, Wakamatsu 2-2, Shinjuku, Tokyo 162-8480, Japan

2 Waseda Institute for Advanced Study, Waseda University, Tokyo, Japan
Keywords Mammalian circadian clock $\cdot$ Liver $\cdot$ Muscle · Oxidative stress

\section{Introduction}

Numerous physiological phenomena in the human body, such as sleep-wake cycles, hormonal and nervous activity, and body temperature, exhibit rhythmic changes over the course of $24 \mathrm{~h}$ (Fig. 1) [1, 2]. These oscillations are regulated by an internal circadian clock system, of which the central pacemaker is located in the suprachiasmatic nucleus (SCN) of the hypothalamus [3, 4]. Peripheral tissues also contain circadian clocks that regulate local physiological functions, and essential core clock genes such as Clock, Bmall, Per1/2, and Cryl/2 have been shown to cooperate with each other to generate cell-autonomous oscillations with circadian rhythm accuracy [1].

In addition, approximately 8-10\% of all genes exhibit rhythmic mRNA expression, which is produced by several important circadian transcriptional factors, including RORs, PPARs, REV-ERBs, SREBPs, DBP, TEF, and HLF $[5,6]$. Recent microarray analysis demonstrated an overlap of these genes in various tissues; however, there are also large disparities in the expression of specific rhythmic genes in each tissue, suggesting that each peripheral clock regulates tissue-specific functions [7]. Moreover, posttranscriptional and post-translational modifications, which have been observed using recently developed sequencing techniques, are also important factors that affect molecular clocks and clock-regulated functions [8,9].

Circadian clocks are invariably either fixed or are adjusted by external stimuli, including sunlight for "photic entrainment", and food/nutrition, temperature, arousal, stress, and exercise for "non-photic entrainment" (Fig. 1) 


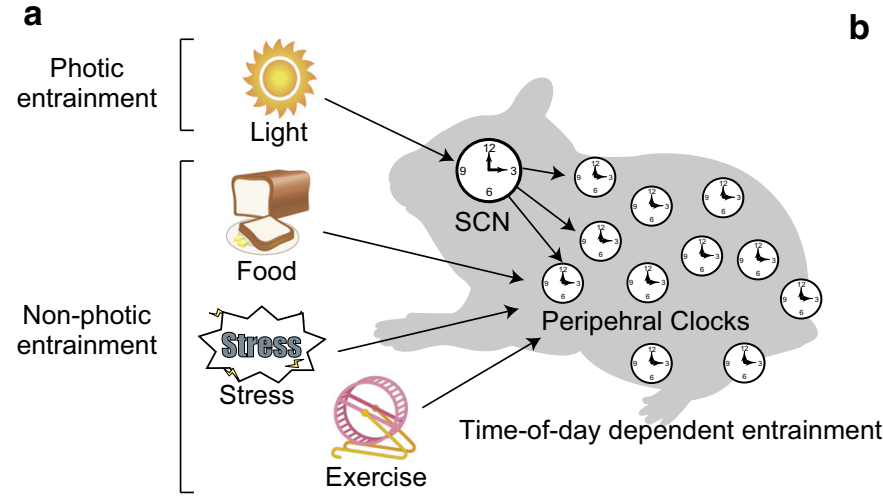

Fig. 1 Schematic diagram of the mammalian circadian clock. a External cues, such as light, food, stress, and exercise, entrain the central (suprachiasmatic nucleus; SCN) and peripheral (peripheral tissues) clocks. Light directly entrains the SCN, whereas other stimuli reset the peripheral clocks, and entrainment depends on the timing of

$[1,2]$. In the case of photic entrainment, light stimuli are conveyed directly to the SCN and transmit principal information to master clocks [10, 11]. Furthermore, light stimuli at the beginning of the active period cause phase delays in the SCN and its associated activity rhythms [10], whereas light stimuli at the end of the active period induce a phase advance of the associated rhythms. Therefore, the effects of external stimuli on the circadian system depend on the time of day (this phenomenon is discussed further in "Time-of-day dependency of non-photic entrainment").

Food also has the potential to entrain peripheral clocks but only has small effects on the master clock, since the light signal is the most important stimulus in the SCN $[12,13]$. Furthermore, scheduled feeding during the inactive period in mice produces changes in sleep-wake cycles, hormonal and temperature rhythms, and the expression of clock genes in peripheral tissues. In addition to light and food, scheduled stress or exercise stimulations have also been reported to be important entrainment factors in mammals.

In this review, we focus on the entrainment of the mammalian circadian clock and discuss the type of stress (e.g., restraint, social defeat, sleep deprivation, or oxidative stress) or exercise (e.g., endurance or resistance exercise), timing of stimulation (e.g., morning or evening), duration of stimulation (e.g., acute or chronic), and signaling pathways (e.g., sympathetic nerve activation or glucocorticoid release). We also review circadian variation in responses to stressor stimuli at different times of the day, as well as the relationship between stress and exercise-induced entrainment. Because both stress and exercise activate the hypothalamic-pituitary-adrenal (HPA) and sympatheticadrenal-medullary (SAM) axes [14], we suggest that these pathways could be involved in entrainment. In addition, we discuss the beneficial effects of exercise on circadian disturbances.

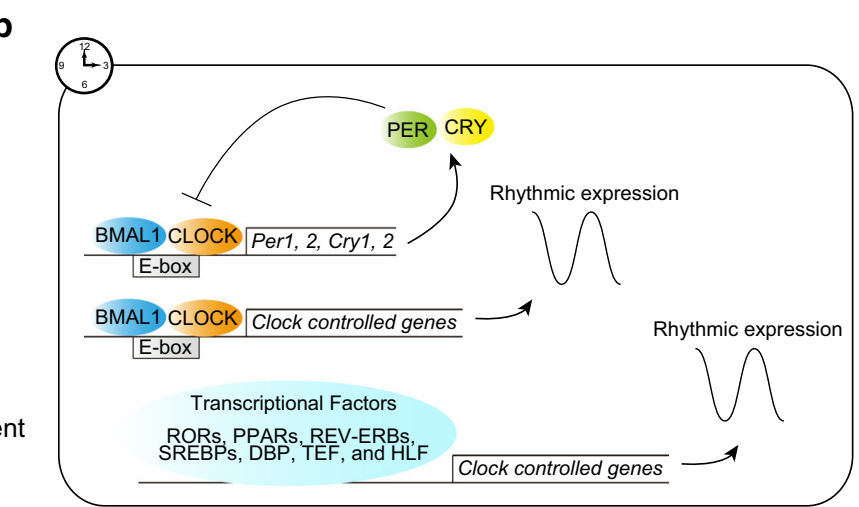

stimulation. b The molecular clock is regulated by transcriptional feedback loops of core clock genes, and oscillations of clockregulated genes in each tissue are controlled by various transcriptional factors, including CLOCK/BMAL1, RORs, PPARs, REV-ERBs, SREBPs, DBP, TEF, and HLF

\section{Stress-induced entrainment of the circadian clock}

\section{Effects of stress and sleep deprivation on the SCN and behavior}

Several studies have shown the phase-resetting effects of acute stress stimuli such as sleep deprivation and social defeat on the sleep-wake cycle of hamsters $[15,16]$. For example, in constant darkness, phase-shifts in locomotor activity rhythms were strongly induced by $3 \mathrm{~h}$ of sleep deprivation that was maintained using gentle handling [15, 16]. In addition, $3 \mathrm{~h}$ of wheel running also caused clear phase entrainment of the behavioral rhythms in hamsters [17], and $3 \mathrm{~h}$ of social defeat stress also caused a phase shift. However, $3 \mathrm{~h}$ of restraint stress did not affect the behavior of hamsters [16], and the treatments that did induce acute phase-shift effects were comparatively small, or not observed in rats [18]. Thus, the type of stimuli and species affected are both important in phase entrainment of the sleep-wake cycle.

The sleep-wake cycle is thought to be the main output rhythm controlled by the SCN clock. In hamsters or mice, for example, sleep deprivation causes rapid reduction in the expression of C-FOS and PER1 proteins and Per1/2 mRNA in the SCN, although the phase entrainment after these changes was not examined [15, 19]. However, in mice, we demonstrated that, under normal light-dark cycles, the SCN clock remains unchanged following 3 days of 2-h restraint stress [20], which suggests that short-term stressors have little effect on the SCN and SCN-regulated activity rhythms in mice. This could be because the glucocorticoid receptor is not expressed in the SCN [21].

However, the SCN clock is sometimes affected by longterm stressors [22-25]. Kinoshita et al. [23], for example, demonstrated that $3 \mathrm{~h}$ of restraint stress at Zeitgeber time 
(ZT)6-9 for 7 consecutive days produced elevated glycogen synthase kinase (GSK)-3 $\beta$ phosphorylation and blunted PER2 rhythms in the SCN of mice (ZT0 and 12 are defined as the start and end of the light period, respectively). In addition, 19 days of social defeat stress during the day or night induced changes in the expression of Per2 and Cryl in the SCN of mice [22]. Moreover, chronic (4 weeks) mild stress caused decreased amplitude of PER2 rhythms in the SCN of rats, whereas 7 days of mild stress caused no alterations [25]. Thus, the sleep-wake cycle and the SCN clock could be entrained or manipulated by stressful stimuli; however, the effects are dependent on the duration and type of stimuli, as well as on the animals affected.

\section{Effect of stress on peripheral circadian clocks}

We recently reported that both acute and sub-acute physiological/psychological stress have tremendous potential to entrain the phases of peripheral circadian clocks in mice, similar to food-induced entrainment (Fig. 2) [20]. We demonstrated that several days of restraint stress or social defeat stress could cause strong phase changes in the PER2::LUC bioluminescence rhythms of the liver, kidney, and submandibular gland in mice and that the effects of the stimuli depended on the time of day and varied with the length (number of days) of stimulation [20]. In fact, we demonstrated that 3 days of restraint stress at ZT4-6 caused a phase advance (4-6 h) of peripheral PER2::LUC rhythms (Fig. 2) [20]. Furthermore, we also determined the stress-induced entrainment of the adrenal gland, cortex, and hippocampus and found that, in contrast to peripheral tissues, the SCN was not affected [20].

Other previous studies have attempted to identify the effect of stressful stimuli on the peripheral clocks in depression models of mice and rats; however, only small phase changes were observed, with no reduction in amplitude. For example, Takahashi et al. [25] demonstrated that 7 days of chronic mild stress induced phase a
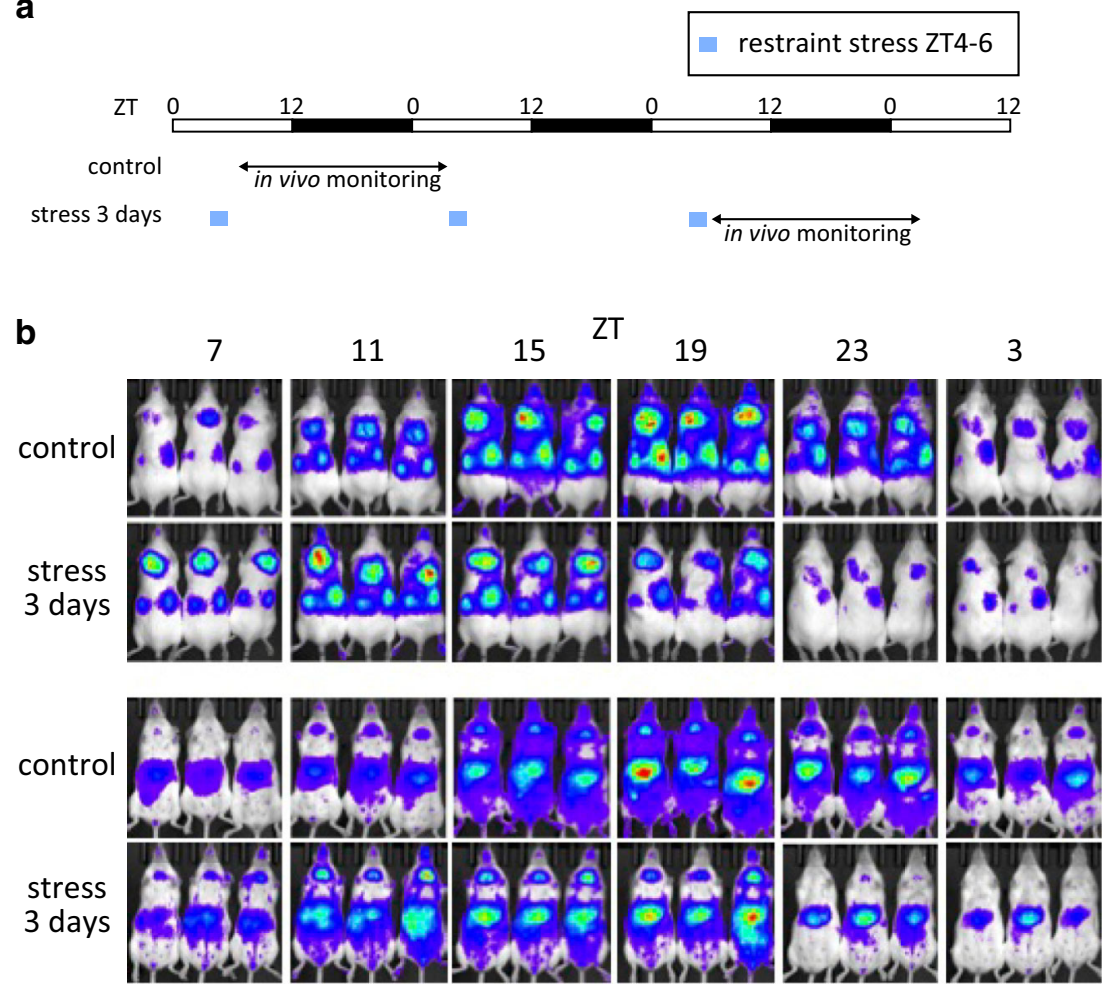
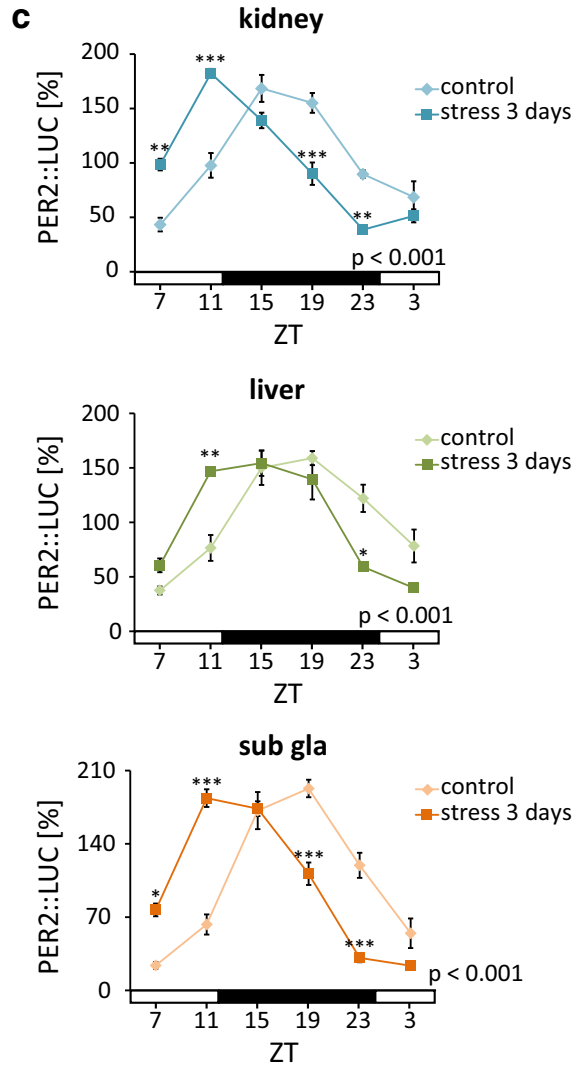

Fig. 2 Stress-induced phase shift of peripheral PER2::LUC rhythms. a Experimental schedule; 2-h restraint stress was performed for 3 days at Zeitgeber time (ZT)4-6 in PER2::LUC mice and, subsequently, the rhythm of in vivo bioluminescence was monitored. b Representative images of in vivo PER2::LUC bioluminescence in kidney (upper panels), liver, and submandibular gland (sub gla) tissues (lower panels). c Normalized PER2::LUC oscillations in control and stress groups show phase advancement in the stressed group. Values are expressed as mean \pm SEM. The $P$ values shown on the lower right side of the graphs indicate the results of two-way ANOVA (with Tukey post hoc test) between the control and stress groups. $* P<0.05, * * * P<0.001$ (modified from [16]) 
advancement of the rhythmic expression of liver clock genes in BALB/c mice; however, the phenomenon was not observed in C57BL/6 mice. Chronic (2-week) daytime social stress was also shown to cause phase advancement (1-2 h) in the PER2::LUC rhythms of cultured adrenal and pituitary glands [24], and in our most recent study, we found that 4 weeks of restraint stress ( 3 days week $^{-1}$ ) elicited habituated responses in the phase entrainment of peripheral PER2::LUC rhythms, with no reductions in amplitude [20]. Thus, it seems that the phases of peripheral clocks could generally be changed by acute or sub-acute stressful stimuli, although habituation to chronic stressful stimuli can reduce these effects.

\section{Entrainment pathways of stress-induced phase changes}

The pathways involved in stress-induced entrainment of peripheral clocks have also been investigated, and along the HPA axis, glucocorticoids, which are secreted from the adrenal gland in response to stressful stimuli, have been identified as powerful factors, both in vitro and in vivo [21]. Functional glucocorticoid response elements (GREs) in the promoter regions of Perl, Per2, and E4bp 4 have also been reported as possible factors in the signaling mechanisms of molecular clocks [26, 27], and the expression of Rev-erb $\alpha$ in the liver has been reported to decrease in response to glucocorticoid treatment via GREs [28]. In our most recent study, we also confirmed that dexamethasone (an analog of corticosterone) induced phase entrainment of peripheral PER2 rhythms in the liver, kidney, and submandibular gland [20]. Restraint stress-induced Perl expression in the liver through GRE has also been reported [29], and the ablation of glucocorticoid effects via adrenalectomy was shown to disrupt PER2 oscillations in the bed nucleus of the stria terminalis (BNST) [30], suggesting that adrenal hormones play an important role in maintaining appropriate circadian rhythms in peripheral tissues in vivo.

Furthermore, sympathetic activation of the SAM axis during stressful stimulation also causes phase changes in peripheral clocks [20], and the administration of adrenaline or noradrenaline induces Perl and Per2 expression through the cAMP response element-binding protein (CREB) signaling pathway [31-33]. Thus, the SAM axis is another pathway involved in stress-induced peripheral clock entrainment.

In addition, restraint stress also induces strong expression of the $\mathrm{HO}-\mathrm{l}$ gene and causes oxidative stress by reducing levels of superoxide dismutase (SOD), glutathione- $S$-transferase (GST), and catalase [34]. Because cellular oxidative stress from the administration of $\mathrm{H}_{2} \mathrm{O}_{2}$ has been reported to reset the expression of clock genes in vitro [35], it is also likely that oxidative stress, as a consequence of physiological stress, could be one of the important pathways in stress-induced phase shifts in vivo. Therefore, several pathways, including the HPA and SAM axes and oxidative stress, may all be involved in the regulation of stress-induced peripheral clock entrainment.

\section{Time-of-day dependency of non-photic entrainment}

The construction of phase response curves (PRCs), in which phase shift values are plotted against the timing of stimuli, is helpful for understanding the properties of entraining stimuli. In a previous study [20], for example, we constructed the PRC of restraint stress-induced peripheral PER2::LUC entrainment and found that phase and amplitude changes were dependent on time of day (Fig. 3): stress at ZT4-6 caused phase-advancement (positive shifts) and stress at ZT18-20 caused phase-delay (negative shifts).

Interestingly, we also found that stress at ZT0-2 caused desynchronization of PER2::LUC among tissues and decreased PER2::LUC amplitude in the kidney (Fig. 3b). This phenomenon in the kidney has been previously attributed to singularity behavior, which constitutes a potent entraining stimulus delivered at the critical transition from phase-delay to phase-advancement, resulting in desynchronization of individual cellular clocks [36-38]. Ukai et al. [37] also observed this phenomenon (i.e., stopping the oscillation of clock gene expression) in melanopsin-transfected NIH3T3 cells in vitro and in SCN clocks in vivo, in response to light perturbation. The PRC of stress entrainment follows a similar trend to that of lightinduced singularity, since ZT0-2 is the transition phase of the PRC (Fig. 3).

In addition, we recently constructed the PRC of caffeine-induced peripheral PER2::LUC phase entrainment in vivo and demonstrated that similar singularity behaviors occur in the liver, kidney, and submandibular gland, as a result of caffeine injections at ZT1 (Fig. 4) [39]. Administration of caffeine induces potent arousal through adenosine receptors. Thus, non-photic entrainment might be induced by caffeine injections. However, for dexamethasone-induced entrainment, Balsalobre et al. [21] constructed the PRC of liver $D b p$ expression rhythms and found no singularity phenotypes at the transition phase, since singularity could be shown by an infinitesimal range of perturbation strengths and timing [37]. Therefore, the construction of PRCs that represent various timings and strengths of stimuli will be necessary to further understand the entraining stimuli of the circadian clock system. 

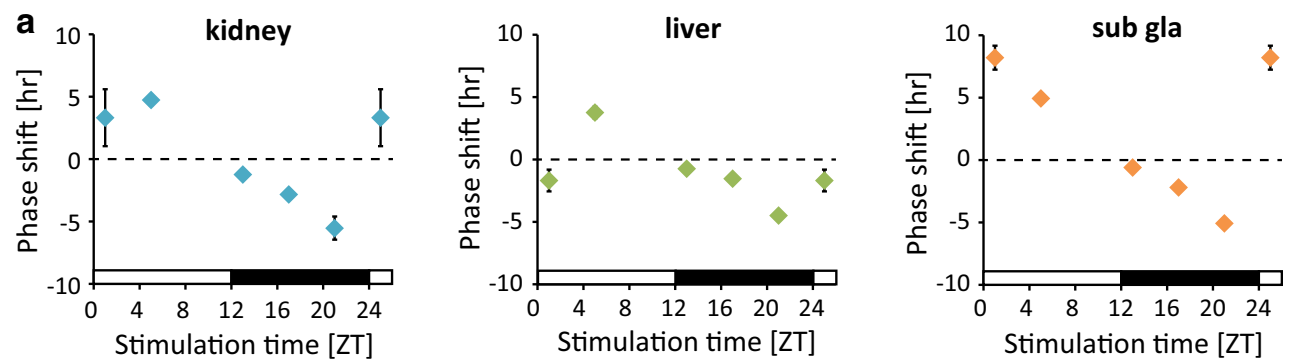

b
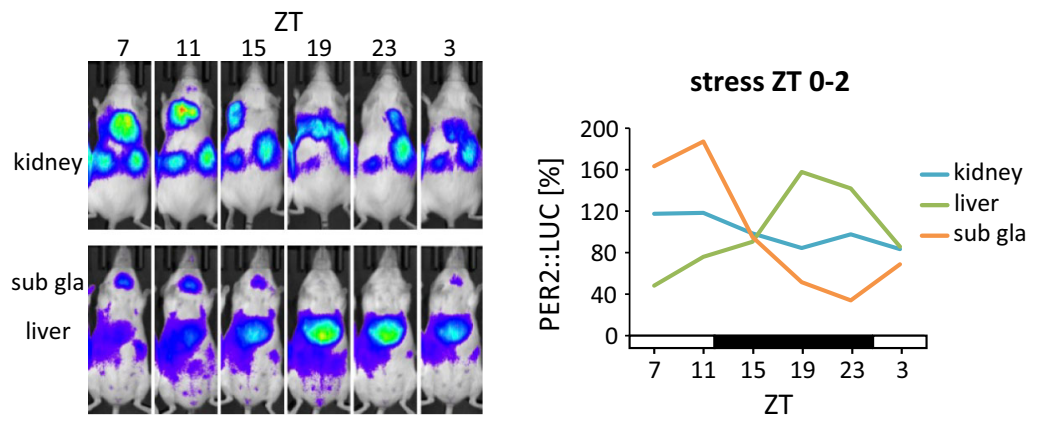

Fig. 3 Time-of-day dependence of circadian changes in response to restraint stress. a Phase-response curves of the response of peripheral clocks to 2-h restraint stress at Zeitgeber time (ZT)0-2, 4-6, 12-14, 16-18, and 20-22 (PER2::LUC rhythms). Increased and decreased phase shifts indicate phase-advance and -delay, respectively. Data for

ZT25 were copied from ZT1. Graphs include all rhythmic and arrhythmic data. b Representative images of in vivo PER2::LUC bioluminescence (left) and normalized waveforms (right) after restraint stress at ZT0-2 for 3 days (modified from [16])

a

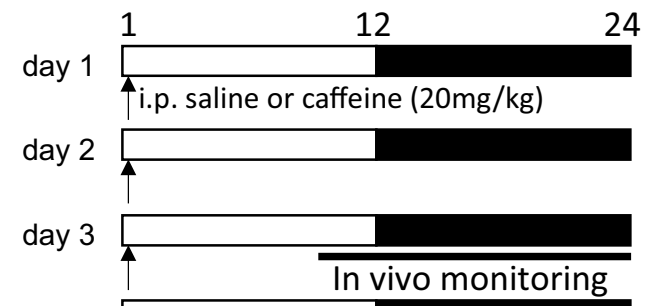

day 4
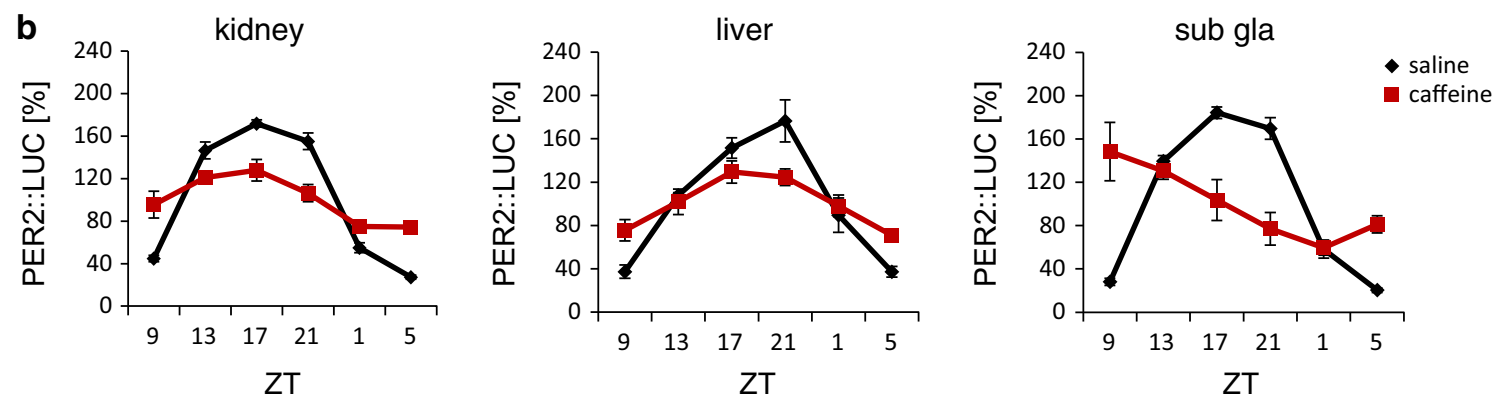

Fig. 4 Decreased amplitude of PER2::LUC rhythms in peripheral tissues after daily caffeine injections. a Experimental schedule; mice were maintained under 12:12 h light-dark conditions and given intraperitoneal (IP) injections of saline (control; VEH) or $20 \mathrm{mg} \mathrm{kg}^{-1}$

caffeine (CAF) for 3 consecutive days at Zeitgeber time (ZT)1; in vivo monitoring was initiated at ZT9. b Normalized waveforms of PER2::LUC rhythms in saline- or caffeine-injected mice 


\section{Circadian clock regulation in the stress response}

\section{Circadian regulation of the HPA axis}

The HPA axis is regulated by the circadian clock system [40]. Elevated levels of circulating glucocorticoids have been observed at the end of the resting phase and are thought to be released in preparation for waking up in the morning. The HPA axis comprises endocrine negative feedback loops that involve the neuropeptides corticotropin-releasing hormone and arginine vasopressin in the paraventricular nucleus (PVN), adrenocorticotropic hormone (ACTH) from the pituitary, and glucocorticoids from the adrenal glands. Each phase of the HPA axis is controlled by circadian rhythms. For example, the expression of $c$-Fos (a marker of neural activity) in the PVN exhibits circadian changes, with high expression levels at the beginning of the dark phase in mice [41, 42]. In addition, serum ACTH and corticosterone concentrations exhibit similar circadian oscillations. Moreover, since these rhythms were not observed in animals with SCN lesions $[43,44]$ or in knockout $(\mathrm{KO})$ mice with clock gene mutations [45, 46], the HPA axis may be regulated by the master clock.

However, Son et al. [47] demonstrated that knockdown of the adrenal-specific Bmall in mice resulted in disrupted rhythms of corticosterone production, via rhythmic expression of the StAR gene in the adrenal gland, suggesting that local clock genes are also important regulators of the circadian cycle of the HPA axis. Furthermore, these mice exhibited abnormal locomotor activity and abnormal expression of clock genes in other peripheral tissues, which suggests that the adrenal clock moderates time-related communication among tissues [47]. However, adrenalectomized mice exhibit no alteration in the expression of clock genes in the liver [48, 49].

Although circadian rhythms of basal corticosterone circulation exist, restraint stress-induced corticosterone levels are similar, whether in response to daytime or nighttime stress [42]. Therefore, the ratio of corticosterone release in response to stress is higher at the beginning of the light phase than at the onset of the dark phase. In addition, Bmall KO mice exhibit lower levels of corticosterone induction in response to restraint stress and lower feedback activity of dexamethasone-induced suppression of corticosterone secretion than wild type (WT) mice. Bmall KO mice also display manic-like behavior in the forced swim test [45]. Thus, the hypothalamic and adrenal clocks regulate both the HPA axis and the stress response.

Furthermore, dysfunction of the HPA axis induces mood spectrum disorders, such as major depression [50]. Rotat- ing nighttime shift work, for example, increases the risk of depressive symptoms [51, 52], and in the mouse model of chronic jet lag, exposure to a 7-h light-dark cycle ( $3.5 \mathrm{~h}: 3.5 \mathrm{~h}=$ light:dark) caused depressive behavior with increased serum corticosterone, demonstrating that disrupted sleep-wake cycles are linked to mood disorders.

However, in rodents, clock gene mutations (e.g., Bmall KO mice and Per2 or Clock mutants) cause hyperactivity, manic-like behavior, and low anxiety that could be attributed to an increase in the dopaminergic activity of the ventral tegmental area (VTA) via changes in the genes for tyrosine hydroxylase and monoamine oxidase A [45, 5355]. In addition, changes in the genes for tyrosine hydroxylase and monoamine oxidase $\mathrm{A}$ also increase the risk of cocaine addiction [56]. Therefore, impairments in the expression of clock genes are, in turn, linked to antidepressive behavior.

On the other hand, there are many reports of singlenucleotide polymorphisms (SNPs) found in clock genes of patients with mood spectrum disorders, such as bipolar disorder, unipolar disorder, and seasonal affective disorder [57]. Thus, further research is required to investigate the relationship between moods and the circadian clock.

\section{Circadian regulation of oxidative stress}

Responses of the antioxidant pathway to oxidative stress also exhibited day-night differences. For example, the severity of bleomycin-induced lung fibrosis in mice exhibited an association with the timing of treatment; fibrosis was more severe in mice treated at ZT12 than in those treated at ZT0 [58]. This was owing to circadian variation of a key antioxidant regulator, nuclear factor erythroid-derived 2-like 2 (Nrf2), which is regulated by CLOCK/BMAL1 through the E-box [58, 59]. Miura et al. [60] also demonstrated that cadmium-induced mortality is affected by circadian variations, since the toxicity of cadmium was higher at ZT8 than at ZT20, and hepatic glutathione (GSH) was lower at ZT8 than at ZT20.

In addition to $N r f 2$ and GSH, many other antioxidants and antioxidant genes exhibit day-night fluctuations as well, including glutathione $S$-transferases, cyclooxygenase2, catalase, and hepatic metallothionein [61-63]. However, the disruption of circadian systems in Bmall KO mice results in increased levels of reactive oxygen species in peripheral tissues, compared to the levels in WT mice, and accelerates aging [64]. Thus, the circadian clock system regulates the responses of oxidative stressors by regulating antioxidant pathways. 


\section{Exercise and the circadian clocks}

\section{Exercise and the entrainment of the circadian clocks}

Exercise represents another non-photic phase-shifting cue that entrains circadian clocks. Some studies have shown that exercise shifts the phase of circadian rhythm of wheel running behavior in rodents under constant dark conditions [17, 65]. For example, Maywood et al. [19] reported that, under constant dark conditions, the expression of Perl and Per2 in the SCN was changed by wheel running.

In addition, the timing of exercise is also involved the regulation of circadian clocks. Wheel running at the onset of the active phase decreases the amplitude of Per2 in the SCN more than that at the end of the active phase [66], and scheduled exercise can entrain the molecular clocks of skeletal muscle and lungs, but not the SCN, under lightdark conditions [67]. Recently, we also demonstrated scheduled exercise-induced entrainment of Per 2 in the submandibular gland [68]. These results suggest that scheduled exercise entrains the molecular clock in both the SCN and peripheral tissues, although exercise-induced entrainment of the master clock is limited under non-light conditions.

Similar to the studies in mice and rats, exercise-induced phase shifts of the circadian rhythm have also been observed in humans [69-74]. For example, Barger et al. [69] reported that exercise accelerated forced sleep-induced phase delays of circadian rhythms in humans. During their study, the daily rhythm of plasma melatonin levels was used as a parameter of circadian rhythms, and the 9-h sleep schedule-induced phase delay of the circadian melatonin rhythm was facilitated by bicycle ergometer exercise under dim light conditions.

Yamanaka et al. [75] also reported that exercise had differential effects on the circadian melatonin rhythm and the sleep-wake cycle. Apparently, exercise accelerated the re-entrainment of the sleep-wake cycle, but not the melatonin rhythm, under dim light and a restricted phase-advanced sleep schedule [75]. Moreover, Yamanaka et al. [74] investigated the effects of exercise on the circadian melatonin rhythm and sleep-wake cycle under bright light and 8-h phase-advance shifted sleep-wake schedule. The sleep-wake cycle was entrained by the sleep schedule, regardless of the presence of exercise, but phase-advancement of the circadian rhythm of melatonin was dependent on exercise. Thus, the combination of light and exercise is a strong entrainment cue for circadian rhythms in humans.

Zambon et al. reported that resistance exercise changed the expression of the molecular clock in human skeletal muscle [76]. The resistance exercise (ten sets of eight repetitions of isotonic knee extension at $80 \%$ of the predetermined one-repetition maximum) changed the gene expression of circadian clocks in the skeletal muscle of humans, suggesting that both resistance and endurance exercise are capable of producing phase shifts in circadian genes of skeletal muscle.

\section{Entrainment pathways of exercise-induced phase changes}

The potential pathways involved in exercise-induced entrainment of peripheral clocks includes the HPA and SAM axes, since several studies have reported their activation by both exercise and stress [77-81]. However, plasma corticosterone levels exhibit day-to-night fluctuations and are highest at the end of the resting phase. Some reports have demonstrated that wheel running only increases corticosterone levels at the end of the resting phase [82, 83], and Fediuc et al. [78] reported that sustained exercise gradually reduced both corticosterone releases.

It is thought that central and peripheral catecholamines regulate the exercise-induced elevation of plasma corticosterone [84]. Although the stress-induced elevation of plasma corticosterone is thought to involve ACTH secretion, the elevation of ACTH is not observed during voluntary exercise, such as wheel running. However, stressful exercise, such as treadmill running, has been shown to increase both ACTH and stress levels in rodents. These findings suggest that the mechanisms of exercise-induced elevations in corticosterone levels differ depending on the type and intensity of the exercise.

\section{Exercise and circadian disturbance}

Some studies have demonstrated beneficial effects of exercise on circadian disturbance. For example, skeletal muscle in Clock mutant mice exhibits decreased mitochondrial content and exercise intolerance [85]; however, endurance exercise for 8 weeks increases skeletal muscle mitochondrial levels and exercise tolerance. Schroeder et al. [66] also reported that the rhythmic deficits observed in vasointestinal polypeptide (VIP)-deficient mice were improved by wheel running. The VIP-deficient mice displayed advanced phases in activity, heart rate, and body temperature rhythms, and a decreased amplitude of Per2 expression in the SCN.

However, the VIP-deficient mice only improved in response to wheel running at the end of the active phase, not at its onset [66], and we recently demonstrated that wheel running at the end of the active phase controlled high-fat diet-induced obesity in mice more than at the onset 
of the active phase [86]. Therefore, although much research has focused on the beneficial effects of exercise itself, the work of Schroeder et al. and ourselves has demonstrated the importance of exercise timing in the resolution of circadian disturbance and metabolic disorder [66, 86].

\section{Conclusions and perspectives}

Based on recent findings, stress and exercise are potent entraining cues for peripheral clocks and sometimes for the central clock. The disturbance of circadian rhythms occurs in several disorders such as cardiovascular disease, obesity, and diabetes [87]. Recent studies have also shown that scheduled feeding can enhance the circadian oscillations of clock genes and metabolic genes, potentially conferring tolerance to high-fat diet-induced obesity and age-related cardiovascular failure [88, 89]. These findings suggest that the timing of meals is an important factor for good health. In addition, exercise may also be a beneficial and powerful tool for the maintenance of circadian rhythms and good health. However, further evidence is needed.

Acknowledgments This work was partially supported by the Council for Science, Technology, and Innovation; SIP; and Technologies for Creating Next-generation Agriculture, Forestry, and Fisheries (funding agency: Bio-oriented Technology Research Advancement Institution, NARO); as well as by a Grant-in-Aid for Scientific Research (S; 26220201) and Grant-in-Aid for Young Scientists (B; 15K18981) from the Ministry of Education, Culture, Sports, Science, and Technology of Japan.

\section{Compliance with ethical standards}

Conflict of interest The authors declare no competing financial interests.

\section{References}

1. Bass J, Takahashi JS (2010) Circadian integration of metabolism and energetics. Science 330(6009):1349-1354

2. Tahara Y, Shibata S (2013) Chronobiology and nutrition. Neuroscience 253:78-88

3. Moore RY, Eichler VB (1972) Loss of a circadian adrenal corticosterone rhythm following suprachiasmatic lesions in the rat. Brain Res 42(1):201-206

4. Tahara Y, Kuroda H, Saito K, Nakajima Y, Kubo Y, Ohnishi N, Seo Y, Otsuka M, Fuse Y, Ohura Y, Komatsu T, Moriya Y, Okada S, Furutani N, Hirao A, Horikawa K, Kudo T, Shibata S (2012) In vivo monitoring of peripheral circadian clocks in the mouse. Curr Biol 22(11):1029-1034

5. Panda S, Antoch MP, Miller BH, Su AI, Schook AB, Straume M, Schultz PG, Kay SA, Takahashi JS, Hogenesch JB (2002) Coordinated transcription of key pathways in the mouse by the circadian clock. Cell 109(3):307-320

6. Ueda HR, Chen W, Adachi A, Wakamatsu H, Hayashi S, Takasugi T, Nagano M, Nakahama K, Suzuki Y, Sugano S, Iino M, Shigeyoshi Y, Hashimoto S (2002) A transcription factor response element for gene expression during circadian night. Nature 418(6897):534-539

7. Zhang R, Lahens NF, Ballance HI, Hughes ME, Hogenesch JB (2014) A circadian gene expression atlas in mammals: implications for biology and medicine. Proc Natl Acad Sci USA 111(45):16219-16224

8. Koike N, Yoo SH, Huang HC, Kumar V, Lee C, Kim TK, Takahashi JS (2012) Transcriptional architecture and chromatin landscape of the core circadian clock in mammals. Science 338(6105):349-354

9. Menet JS, Rodriguez J, Abruzzi KC, Rosbash M (2012) NascentSeq reveals novel features of mouse circadian transcriptional regulation. Elife 1:e00011

10. Colwell CS (2011) Linking neural activity and molecular oscillations in the SCN. Nat Rev Neurosci 12(10):553-569

11. Colwell CS, Foster RG (1992) Photic regulation of Fos-like immunoreactivity in the suprachiasmatic nucleus of the mouse. J Comp Neurol 324(2):135-142

12. Acosta-Galvan G, Yi CX, van der Vliet J, Jhamandas JH, Panula P, Angeles-Castellanos M, Del Carmen Basualdo M, Escobar C, Buijs RM (2011) Interaction between hypothalamic dorsomedial nucleus and the suprachiasmatic nucleus determines intensity of food anticipatory behavior. Proc Natl Acad Sci USA 108(14):5813-5818

13. Hara R, Wan K, Wakamatsu H, Aida R, Moriya T, Akiyama M, Shibata S (2001) Restricted feeding entrains liver clock without participation of the suprachiasmatic nucleus. Genes Cells 6(3):269-278

14. Mastorakos G, Pavlatou M, Diamanti-Kandarakis E, Chrousos GP (2005) Exercise and the stress system. Hormones (Athens) 4(2):73-89

15. Antle MC, Mistlberger RE (2000) Circadian clock resetting by sleep deprivation without exercise in the Syrian hamster. J Neurosci 20(24):9326-9332

16. Mistlberger RE, Antle MC, Webb IC, Jones M, Weinberg J, Pollock MS (2003) Circadian clock resetting by arousal in Syrian hamsters: the role of stress and activity. Am J Physiol Regul Integr Comp Physiol 285(4):R917-R925

17. Reebs SG, Mrosovsky N (1989) Effects of induced wheel running on the circadian activity rhythms of Syrian hamsters: entrainment and phase response curve. J Biol Rhythms 4(1):39-48

18. Barrington J, Jarvis H, Redman JR, Armstrong SM (1993) Limited effect of three types of daily stress on rat free-running locomotor rhythms. Chronobiol Int 10(6):410-419

19. Maywood ES, Mrosovsky N, Field MD, Hastings MH (1999) Rapid down-regulation of mammalian period genes during behavioral resetting of the circadian clock. Proc Natl Acad Sci USA 96(26):15211-15216

20. Tahara Y, Shiraishi T, Kikuchi Y, Haraguchi A, Kuriki D, Sasaki H, Motohashi H, Sakai T, Shibata S (2015) Entrainment of the mouse circadian clock by sub-acute physical and psychological stress. Sci Rep 5:11417

21. Balsalobre A, Brown SA, Marcacci L, Tronche F, Kellendonk C, Reichardt HM, Schutz G, Schibler U (2000) Resetting of circadian time in peripheral tissues by glucocorticoid signaling. Science 289(5488):2344-2347

22. Bartlang MS, Savelyev SA, Johansson AS, Reber SO, HelfrichForster C, Lundkvist GB (2014) Repeated psychosocial stress at night, but not day, affects the central molecular clock. Chronobiol Int 31(9):996-1007

23. Kinoshita C, Miyazaki K, Ishida N (2012) Chronic stress affects PERIOD2 expression through glycogen synthase kinase-3beta phosphorylation in the central clock. Neuroreport 23(2):98-102

24. Razzoli M, Karsten C, Yoder JM, Bartolomucci A, Engeland WC (2014) Chronic subordination stress phase advances adrenal and 
anterior pituitary clock gene rhythms. Am J Physiol Regul Integr Comp Physiol 307(2):R198-R205

25. Takahashi K, Yamada T, Tsukita S, Kaneko K, Shirai Y, Munakata Y, Ishigaki Y, Imai J, Uno K, Hasegawa Y, Sawada S, Oka Y, Katagiri H (2013) Chronic mild stress alters circadian expressions of molecular clock genes in the liver. Am J Physiol Endocrinol Metab 304(3):E301-E309

26. Cheon S, Park N, Cho S, Kim K (2013) Glucocorticoid-mediated Period 2 induction delays the phase of circadian rhythm. Nucleic Acids Res 41(12):6161-6174

27. So AY, Bernal TU, Pillsbury ML, Yamamoto KR, Feldman BJ (2009) Glucocorticoid regulation of the circadian clock modulates glucose homeostasis. Proc Natl Acad Sci USA 106(41):17582-17587

28. Torra IP, Tsibulsky V, Delaunay F, Saladin R, Laudet V, Fruchart JC, Kosykh V, Staels B (2000) Circadian and glucocorticoid regulation of Rev-erbalpha expression in liver. Endocrinology 141(10):3799-3806

29. Yamamoto T, Nakahata Y, Tanaka M, Yoshida M, Soma H, Shinohara K, Yasuda A, Mamine T, Takumi T (2005) Acute physical stress elevates mouse period1 mRNA expression in mouse peripheral tissues via a glucocorticoid-responsive element. J Biol Chem 280(51):42036-42043

30. Segall LA, Perrin JS, Walker CD, Stewart J, Amir S (2006) Glucocorticoid rhythms control the rhythm of expression of the clock protein, Period2, in oval nucleus of the bed nucleus of the stria terminalis and central nucleus of the amygdala in rats. Neuroscience 140(3):753-757

31. Akiyama M, Minami Y, Nakajima T, Moriya T, Shibata S (2001) Calcium and pituitary adenylate cyclase-activating polypeptide induced expression of circadian clock gene mPer1 in the mouse cerebellar granule cell culture. J Neurochem 78(3):499-508

32. Terazono H, Mutoh T, Yamaguchi S, Kobayashi M, Akiyama M, Udo R, Ohdo S, Okamura H, Shibata S (2003) Adrenergic regulation of clock gene expression in mouse liver. Proc Natl Acad Sci USA 100(11):6795-6800

33. Travnickova-Bendova Z, Cermakian N, Reppert SM, SassoneCorsi P (2002) Bimodal regulation of mPeriod promoters by CREB-dependent signaling and CLOCK/BMAL1 activity. Proc Natl Acad Sci USA 99(11):7728-7733

34. Zaidi SM, Al-Qirim TM, Hoda N, Banu N (2003) Modulation of restraint stress induced oxidative changes in rats by antioxidant vitamins. J Nutr Biochem 14(11):633-636

35. Tamaru T, Hattori M, Ninomiya $Y$, Kawamura G, Vares G, Honda K, Mishra DP, Wang B, Benjamin I, Sassone-Corsi P, Ozawa T, Takamatsu K (2013) ROS stress resets circadian clocks to coordinate pro-survival signals. PLoS One 8(12):e82006

36. An S, Harang R, Meeker K, Granados-Fuentes D, Tsai CA, Mazuski C, Kim J, Doyle FJ 3rd, Petzold LR, Herzog ED (2013) A neuropeptide speeds circadian entrainment by reducing intercellular synchrony. Proc Natl Acad Sci USA 110(46):E4355-E4361

37. Ukai H, Kobayashi TJ, Nagano M, Masumoto KH, Sujino M, Kondo T, Yagita K, Shigeyoshi Y, Ueda HR (2007) Melanopsindependent photo-perturbation reveals desynchronization underlying the singularity of mammalian circadian clocks. Nat Cell Biol 9(11):1327-1334

38. Winfree AT (1975) Unclocklike behaviour of biological clocks. Nature 253(5490):315-319

39. Narishige S, Kuwahara M, Shinozaki A, Okada S, Ikeda Y, Kamagata M, Tahara Y, Shibata S (2014) Effects of caffeine on circadian phase, amplitude and period evaluated in cells in vitro and peripheral organs in vivo in PER2:LUCIFERASE mice. Br J Pharmacol 171(24):5858-5869

40. Leliavski A, Dumbell R, Ott V, Oster H (2015) Adrenal clocks and the role of adrenal hormones in the regulation of circadian physiology. J Biol Rhythms 30(1):20-34
41. Cascio CS, Shinsako J, Dallman MF (1987) The suprachiasmatic nuclei stimulate evening $\mathrm{ACTH}$ secretion in the rat. Brain Res 423(1-2):173-178

42. Girotti M, Weinberg MS, Spencer RL (2007) Differential responses of hypothalamus-pituitary-adrenal axis immediate early genes to corticosterone and circadian drive. Endocrinology 148(5):2542-2552

43. Akana SF, Cascio CS, Du JZ, Levin N, Dallman MF (1986) Reset of feedback in the adrenocortical system: an apparent shift in sensitivity of adrenocorticotropin to inhibition by corticosterone between morning and evening. Endocrinology 119(5):2325-2332

44. Szafarczyk A, Ixart G, Malaval F, Nouguier-Soule J, Assenmacher I (1979) Effects of lesions of the suprachiasmatic nuclei and of $p$-chlorophenylalanine on the circadian rhythms of adrenocorticotrophic hormone and corticosterone in the plasma, and on locomotor activity of rats. J Endocrinol 83(1):1-16

45. Leliavski A, Shostak A, Husse J, Oster H (2014) Impaired glucocorticoid production and response to stress in Arntl-deficient male mice. Endocrinology 155(1):133-142

46. Takita E, Yokota S, Tahara Y, Hirao A, Aoki N, Nakamura Y, Nakao A, Shibata S (2013) Biological clock dysfunction exacerbates contact hypersensitivity in mice. $\mathrm{Br} \mathbf{J}$ Dermatol 168(1):39-46

47. Son GH, Chung S, Choe HK, Kim HD, Baik SM, Lee H, Lee HW, Choi S, Sun W, Kim H, Cho S, Lee KH, Kim K (2008) Adrenal peripheral clock controls the autonomous circadian rhythm of glucocorticoid by causing rhythmic steroid production. Proc Natl Acad Sci USA 105(52):20970-20975

48. Ikeda Y, Sasaki H, Ohtsu T, Shiraishi T, Tahara Y, Shibata S (2015) Feeding and adrenal entrainment stimuli are both necessary for normal circadian oscillation of peripheral clocks in mice housed under different photoperiods. Chronobiol Int 32(2):195-210

49. Oishi K, Amagai N, Shirai H, Kadota K, Ohkura N, Ishida N (2005) Genome-wide expression analysis reveals 100 adrenal gland-dependent circadian genes in the mouse liver. DNA Res 12(3):191-202

50. Antonijevic I (2008) HPA axis and sleep: identifying subtypes of major depression. Stress 11(1):15-27

51. Lee HY, Kim MS, Kim O, Lee IH, Kim HK (2016) Association between shift work and severity of depressive symptoms among female nurses: the Korea Nurses' Health Study. J Nurs Manag 24(2): 192-200

52. Scott AJ, Monk TH, Brink LL (1997) Shiftwork as a risk factor for depression: a pilot study. Int $\mathrm{J}$ Occup Environ Health 3(Supplement 2):S2-S9

53. Hampp G, Ripperger JA, Houben T, Schmutz I, Blex C, PerreauLenz S, Brunk I, Spanagel R, Ahnert-Hilger G, Meijer JH, Albrecht U (2008) Regulation of monoamine oxidase A by circadian-clock components implies clock influence on mood. Curr Biol 18(9):678-683

54. McClung CA (2011) Circadian rhythms and mood regulation: insights from pre-clinical models. Eur Neuropsychopharmacol 21(Suppl 4):S683-S693

55. Roybal K, Theobold D, Graham A, DiNieri JA, Russo SJ, Krishnan V, Chakravarty S, Peevey J, Oehrlein N, Birnbaum S, Vitaterna MH, Orsulak P, Takahashi JS, Nestler EJ, Carlezon WA Jr, McClung C (2007) Mania-like behavior induced by disruption of CLOCK. Proc Natl Acad Sci USA 104(15):6406-6411

56. McClung CA, Sidiropoulou K, Vitaterna M, Takahashi JS, White FJ, Cooper DC, Nestler EJ (2005) Regulation of dopaminergic transmission and cocaine reward by the Clock gene. Proc Natl Acad Sci USA 102(26):9377-9381

57. Etain B, Milhiet V, Bellivier F, Leboyer M (2011) Genetics of circadian rhythms and mood spectrum disorders. Eur Neuropsychopharmacol 21(Suppl 4):S676-S682 
58. Pekovic-Vaughan V, Gibbs J, Yoshitane H, Yang N, Pathiranage D, Guo B, Sagami A, Taguchi K, Bechtold D, Loudon A, Yamamoto M, Chan J, van der Horst GT, Fukada Y, Meng QJ (2014) The circadian clock regulates rhythmic activation of the NRF2/glutathione-mediated antioxidant defense pathway to modulate pulmonary fibrosis. Genes Dev 28(6):548-560

59. Lee J, Moulik M, Fang Z, Saha P, Zou F, Xu Y, Nelson DL, Ma K, Moore DD, Yechoor VK (2013) Bmall and beta-cell clock are required for adaptation to circadian disruption, and their loss of function leads to oxidative stress-induced beta-cell failure in mice. Mol Cell Biol 33(11):2327-2338

60. Miura N, Yanagiba Y, Ohtani K, Mita M, Togawa M, Hasegawa $\mathrm{T}$ (2012) Diurnal variation of cadmium-induced mortality in mice. J Toxicol Sci 37(1):191-196

61. Sani M, Sebai H, Gadacha W, Boughattas NA, Reinberg A, Mossadok BA (2006) Catalase activity and rhythmic patterns in mouse brain, kidney and liver. Comp Biochem Physiol B: Biochem Mol Biol 145(3-4):331-337

62. Xu YQ, Zhang D, Jin T, Cai DJ, Wu Q, Lu Y, Liu J, Klaassen CD (2012) Diurnal variation of hepatic antioxidant gene expression in mice. PLoS One 7(8):e44237

63. Zhang YK, Yeager RL, Klaassen CD (2009) Circadian expression profiles of drug-processing genes and transcription factors in mouse liver. Drug Metab Dispos 37(1):106-115

64. Kondratov RV, Kondratova AA, Gorbacheva VY, Vykhovanets OV, Antoch MP (2006) Early aging and age-related pathologies in mice deficient in BMAL1, the core component of the circadian clock. Genes Dev 20(14):1868-1873

65. Marchant EG, Mistlberger RE (1996) Entrainment and phase shifting of circadian rhythms in mice by forced treadmill running. Physiol Behav 60(2):657-663

66. Schroeder AM, Truong D, Loh DH, Jordan MC, Roos KP, Colwell CS (2012) Voluntary scheduled exercise alters diurnal rhythms of behaviour, physiology and gene expression in wildtype and vasoactive intestinal peptide-deficient mice. J Physiol 590(Pt 23):6213-6226

67. Wolff G, Esser KA (2012) Scheduled exercise phase shifts the circadian clock in skeletal muscle. Med Sci Sports Exerc 44(9): 1663-1670

68. Hamaguchi Y, Tahara Y, Hitosugi M, Shibata S (2015) Impairment of circadian rhythms in peripheral clocks by constant light is partially reversed by scheduled feeding or exercise. J Biol Rhythms 30(6):533-542

69. Barger LK, Wright KP Jr, Hughes RJ, Czeisler CA (2004) Daily exercise facilitates phase delays of circadian melatonin rhythm in very dim light. Am J Physiol Regul Integr Comp Physiol 286(6):R1077-R1084

70. Buxton OM, Frank SA, L'Hermite-Baleriaux M, Leproult R, Turek FW, Van Cauter E (1997) Roles of intensity and duration of nocturnal exercise in causing phase delays of human circadian rhythms. Am J Physiol 273(3 Pt 1):E536-E542

71. Buxton OM, Lee CW, L'Hermite-Baleriaux M, Turek FW, Van Cauter E (2003) Exercise elicits phase shifts and acute alterations of melatonin that vary with circadian phase. Am J Physiol Regul Integr Comp Physiol 284(3):R714-R724

72. Miyazaki T, Hashimoto S, Masubuchi S, Honma S, Honma KI (2001) Phase-advance shifts of human circadian pacemaker are accelerated by daytime physical exercise. Am J Physiol Regul Integr Comp Physiol 281(1):R197-R205

73. Van Reeth O, Sturis J, Byrne MM, Blackman JD, L'HermiteBaleriaux M, Leproult R, Oliner C, Refetoff S, Turek FW, Van Cauter E (1994) Nocturnal exercise phase delays circadian rhythms of melatonin and thyrotropin secretion in normal men. Am J Physiol 266(6 Pt 1):E964-E974

74. Yamanaka Y, Hashimoto S, Masubuchi S, Natsubori A, Nishide SY, Honma S, Honma K (2014) Differential regulation of circadian melatonin rhythm and sleep-wake cycle by bright lights and nonphotic time cues in humans. Am J Physiol Regul Integr Comp Physiol 307(5):R546-R557

75. Yamanaka Y, Hashimoto S, Tanahashi Y, Nishide SY, Honma S, Honma K (2010) Physical exercise accelerates reentrainment of human sleep-wake cycle but not of plasma melatonin rhythm to 8-h phase-advanced sleep schedule. Am J Physiol Regul Integr Comp Physiol 298(3):R681-R691

76. Zambon AC, McDearmon EL, Salomonis N, Vranizan KM, Johansen KL, Adey D, Takahashi JS, Schambelan M, Conklin BR (2003) Time- and exercise-dependent gene regulation in human skeletal muscle. Genome Biol 4(10):R61

77. Chennaoui M, Gomez Merino D, Lesage J, Drogou C, Guezennec CY (2002) Effects of moderate and intensive training on the hypothalamo-pituitary-adrenal axis in rats. Acta Physiol Scand 175(2):113-121

78. Fediuc S, Campbell JE, Riddell MC (2006) Effect of voluntary wheel running on circadian corticosterone release and on HPA axis responsiveness to restraint stress in Sprague-Dawley rats. J Appl Physiol (1985) 100(6):1867-1875

79. Hansen D, Meeusen R, Mullens A, Dendale P (2012) Effect of acute endurance and resistance exercise on endocrine hormones directly related to lipolysis and skeletal muscle protein synthesis in adult individuals with obesity. Sports Med 42(5):415-431

80. Stranahan AM, Lee K, Mattson MP (2008) Central mechanisms of HPA axis regulation by voluntary exercise. Neuromol Med 10(2):118-127

81. Zouhal H, Jacob C, Delamarche P, Gratas-Delamarche A (2008) Catecholamines and the effects of exercise, training and gender. Sports Med 38(5):401-423

82. Otawa M, Arai H, Atomi Y (2007) Molecular aspects of adrenal regulation for circadian glucocorticoid synthesis by chronic voluntary exercise. Life Sci 80(8):725-731

83. Yasumoto Y, Nakao R, Oishi K (2015) Free access to a runningwheel advances the phase of behavioral and physiological circadian rhythms and peripheral molecular clocks in mice. PLoS One 10(1):e0116476

84. Yu XN, Komaki G, Sudo N, Kubo C (2001) Central and peripheral catecholamines regulate the exercise-induced elevation of plasma interleukin 6 in rats. Life Sci 69(2):167-174

85. Pastore S, Hood DA (2013) Endurance training ameliorates the metabolic and performance characteristics of circadian Clock mutant mice. J Appl Physiol (1985) 114(8):1076-1084

86. Sasaki H, Ohtsu T, Ikeda Y, Tsubosaka M, Shibata S (2014) Combination of meal and exercise timing with a high-fat diet influences energy expenditure and obesity in mice. Chronobiol Int 31(9):959-975

87. Morris CJ, Yang JN, Scheer FA (2012) The impact of the circadian timing system on cardiovascular and metabolic function. Prog Brain Res 199:337-358

88. Gill S, Le HD, Melkani GC, Panda S (2015) Time-restricted feeding attenuates age-related cardiac decline in Drosophila. Science 347(6227):1265-1269

89. Hatori M, Vollmers C, Zarrinpar A, DiTacchio L, Bushong EA, Gill S, Leblanc M, Chaix A, Joens M, Fitzpatrick JA, Ellisman MH, Panda S (2012) Time-restricted feeding without reducing caloric intake prevents metabolic diseases in mice fed a high-fat diet. Cell Metab 15(6):848-860 